\title{
Germanica
}

\section{Gunnar Gunnarsson et le « roman du peuplement de l'Islande »}

Gunnar Gunnarsson und der « Roman der Bevölkerung Islands »

\section{Einar Már Jónsson}

\section{OpenEdition}

\section{Journals}

Édition électronique

URL : http://journals.openedition.org/germanica/1256

DOI : 10.4000/germanica.1256

ISSN : 2107-0784

Éditeur

Université de Lille

\section{Édition imprimée}

Date de publication : 1 décembre 1998

Pagination : 43-58

ISBN : 0984-2632F

ISSN : 0984-2632

\section{Référence électronique}

Einar Már Jónsson, «Gunnar Gunnarsson et le « roman du peuplement de l'Islande » », Germanica [En ligne], 23 | 1998, mis en ligne le 26 janvier 2012, consulté le 06 octobre 2020. URL : http:// journals.openedition.org/germanica/1256 ; DOI : https://doi.org/10.4000/germanica.1256

Ce document a été généré automatiquement le 6 octobre 2020.

(c) Tous droits réservés 


\title{
Gunnar Gunnarsson et le « roman du peuplement de l'Islande »
}

Gunnar Gunnarsson und der « Roman der Bevölkerung Islands »

\author{
Einar Már Jónsson
}

1 S'il y a aujourd'hui un grand écrivain islandais au purgatoire, c'est bien Gunnar Gunnarsson. On peut se demander s'il n'a pas été victime d'une situation paradoxale qui l'avait pourtant favorisé au début : être un auteur islandais fortement attaché à ses origines et puisant toute son inspiration dans sa terre natale, mais vivant au Danemark et écrivant en danois. On peut également se demander si le discrédit dans lequel est tombée la mouvance littéraire à laquelle on peut le rattacher, qu'on désigne généralement par l'expression allemande "Blut und Boden », n'a pas rejailli sur lui. En tout cas le fait est là. Malgré son immense réputation dans les années vingt et trente, lorsqu'il était parmi les auteurs Scandinaves les plus lus et les plus traduits, en tout cas dans l'Europe du Nord, et une personnalité culturelle très en vue, il a maintenant disparu au Danemark, sans laisser de traces, et les Islandais, qui possèdent ses œuvres dans des traductions plus ou moins bonnes, le connaissent sans vraiment le lire. Curieusement, les seuls signes d'intérêt pour Gunnar Gunnarsson ces dernières années viennent de la France : l'accueil très positif que la critique et le public français ont récemment fait à deux de ses romans, Le berger de l'Avent et Les oiseaux noirs, indique qu'ils ont passé le test du temps et que vis-à-vis de cet écrivain il y a peut-être une grande injustice à réparer.

2 Dans l'œuvre très variée de Gunnar Gunnarsson, la partie la plus méconnue est certainement ses cinq romans historiques, qui forment un ensemble et représentent en fait moins de la moitié d'un grand projet que l'auteur n'a pas réussi à mener à bien. Né dans l'est de l'Islande en 1889 et arrivé au Danemark à l'âge de 18 ans, fermement décidé à devenir écrivain, Gunnar Gunnarsson a formé tôt dans sa carrière le projet d'écrire une série de romans historiques couvrant toute l'histoire de l'Islande depuis l'origine jusqu'à l'époque contemporaine ${ }^{1}$. Dans son esprit, cette série devait porter le titre d'ensemble « Landnám » (ce mot, qui signifie littéralement « prise des terres », est le terme consacré désignant le peuplement de l'Islande, entre 874 et 930, mais l'auteur 
le comprenait dans un sens beaucoup plus large) et compter une douzaine d'œuvres. Le roman qui inaugurait cette série, Les frères jurés (Edbrødre), est sorti en 1918 : il racontait l'histoire du premier colon norvégien qui s'est installé en Islande en 874, et formait donc véritablement le début de cette fresque romanesque. Ensuite Gunnar Gunnarsson s'est détourné de ce projet pendant une douzaine d'années, pour se consacrer, entre autre, à sa série autobiographique L'église de la montagne (Kirken på bjerget), avant de le reprendre dans les années trente. Bousculant l'ordre chronologique, il publia alors, en 1930, Jon Arason, sur l'époque de la réforme luthérienne et la lutte du dernier évêque catholique contre les autorités danoises en Islande. Puis il revint au Moyen âge avec Terre (Jord), en 1933, qui forme une suite des Frères jurés et raconte la fondation de la république islandaise médiévale, Le Christ-blanc (Hvide-Krist), en 1934, sur la christianisation de l'Islande, et finalement Graamand, en 1936, situé au XII ${ }^{e}$ siècle, sur le début des guerres civiles qui devaient avoir des effets catastrophiques pour le pays.

Ces cinq romans correspondent donc à une partie du projet initial de Gunnar Gunnarsson. Parfois il a pensé inclure d'autres de ses romans dans cette série, notamment Les oiseaux noirs (Svartfugi), publié en 1929. Mais si cette dernière œuvre est " historique » en ce sens que l'action se passe au début du XIX siècle et qu'il s'agit de personnages et d'une affaire criminelle bien réels, elle se distingue pourtant très clairement des cinq autres romans : les événements y ont un caractère purement privé et l'auteur s'intéresse surtout à la psychologie des personnages et à la question philosophique de l'innocence et de la culpabilité, tandis que les cinq romans précédemment cités sont chacun centré autour d'un épisode important et dramatique de l'histoire nationale islandaise, envisagé sous un angle historique. Il vaut donc mieux partir du premier projet de l'auteur et regarder ces cinq romans comme un groupe distinct de ses autres œuvres. Cependant on est obligé de constater que ces œuvres historiques n'ont pas d'unité de ton et de thématique qui les rapprocherait et qui en ferait un véritable cycle romanesque: le style et la technique narratifs sont très différents d'un roman à l'autre et on ne trouve pas de grandes idées d'ensemble dans cette série. C'est peut-être cette absence de véritable projet idéologique, autant que les changements dans la vie de l'auteur, qui l'ont amené à abandonner le genre des romans historiques : après avoir quitté définitivement le Danemark en 1939 et s'être installé en Islande, Gunnar Gunnarsson s'est tourné vers d'autres œuvres, et il a surtout consacré la dernière partie de sa longue vie (il est mort en 1975) à traduire en islandais ses romans écrits en danois.

4 Ce que nous avons dit sur l'ensemble des romans historiques de Gunnar Gunnarsson ne vaut cependant pas pour les deux œuvres, Les frères jurés et Terre, auxquelles nous allons spécialement porter notre attention dans la présente étude. Certes, le style de ces œuvres, séparées d'une quinzaine d'années, est extrêmement différent, on pourrait même dire opposé, mais outre le fait que Terre se présente explicitement comme une suite des Frères jurés et qu'ensemble ces deux romans traitent d'une seule période capitale de l'histoire de l'Islande, la période du peuplement, ces deux œuvres sont unifiées par la même thématique qui les traverse du début jusqu'à la fin. Par rapport à cette puissante unité, on a même l'impression que ces romans ressemblent à une œuvre musicale en deux mouvements qui développeraient la même matière musicale dans des tempos différents, rapide et lent...

5 La thématique des Frères jurés et de Terre est purement historique, liée à cette période précise où des colons venus de Norvège ont peuplé l'Islande et créé un système 
politique, et il est donc nécessaire de l'étudier du point de vue de l'histoire. Pour cela il est intéressant de voir d'abord les principales théories sur Thistoriographie au Danemark, au moment où Gunnar Gunnarsson formait son projet romanesque, et, peutêtre surtout, les théories sur les rapports entre celle-ci et le roman historique.

6 En 1911, l'année avant que Gunnar Gunnarsson ne fasse son entrée sur la scène littéraire danoise, un des plus importants historiens du pays, Kristian Erslev, grand spécialiste de l'époque de la reine Marguerite, publia un petit livre intitulé Ecriture de l'histoire ${ }^{2}$, où il exposait ses conceptions de l'historiographie. Cette œuvre correspondait sans doute à "l'esprit du temps »- la révolte contre le positivisme en histoire qui devait plusieurs années plus tard contribuer à la naissance $d u$ «mouvement des Annales » en France - et elle eut une très forte influence sur toute une génération d'historiens danois, et même plus. Contrairement aux positions fondamentales des principaux théoriciens positivistes de l'histoire, tels que Bernheim en Allemagne et Langlois et Seignobos en France, Erslev voulait établir une distinction radicale entre la science historique et l'historiographie : au lieu d'être une simple mise en forme de la science historique et une présentation de ses résultats, comme l'avaient pensé ces théoriciens, l'historiographie est, selon lui, une discipline indépendante qui a son propre but et suit ses propres lois. La science historique a pour objet l'étude d'un document ou d'un groupe de documents, l'établissement d'un fait précis, une nouvelle interprétation de faits connus etc., et elle aboutit généralement à un article dans une revue savante ou une publication érudite, qui obéissent à des règles rigoureuses. Si l'historiographie s'appuie nécessairement sur la science historique, son but est en revanche tout à fait différent : elle cherche à décrire le passé ou un aspect du passé, à en donner une image aussi vivante et vraie que possible. Pour ce faire, l'historiographie a des règles particulières qui n'appartiennent qu'à elle. Erslev ne les étudie pas spécialement en détail, mais il en évoque quelques éléments :

7 - L'historiographe utilise les résultats de la science historique comme matériau, mais il choisit toujours - sinon l'image du passé serait une confusion dépourvue de sens - et il établit ce choix en fonction du tableau qu'il est en train de faire. En général il omet de rendre compte des recherches ou des arguments qui ont abouti à ces résultats, mais il peut choisir de le faire si cela peut contribuer à compléter ou enrichir le tableau.

- Quel que soit le choix, le matériau que l'historiographe a à sa disposition est toujours lacunaire, et, en tant que tel, il est mort. Il faut que l'historiographe lui-même le rende vivant par sa propre intuition et par son imagination.

- En construisant l'image du passé, l'historiographe suit souvent un chemin diamétralement opposé à celui de la science historique et établit des relations tout à fait différentes entre les détails qu'il décrit.

- Pour que son travail ait quelque valeur, il faut que l'historiographe se laisse guider par des idées fondamentales, par une théorie ou une "vision» historique. Quand il compose son tableau du passé, il a ensuite à sa disposition de nombreux artifices de style et de présentation, qui n'ont rien à faire avec la science historique proprement dite mais qui font partie intégrante de l'historiographie. Mais ces artifices ne doivent jamais être gratuits: il faut qu'ils découlent naturellement de l'idée fondamentale derrière l'œuvre.

8 Cette définition de l'historiographie et de ses règles a des conséquences peut-être inattendues : entre l'historiographie ainsi définie et le roman historique il y a plus que des ressemblances, il y a en fait des parallélismes stricts, car les deux sont des images 
du passé qui obéissent à des règles analogues. Ces conséquences, Erslev ne cherche aucunement à les éviter; au contraire, il insiste sur le rapprochement entre les deux genres. Il souligne ainsi que l'auteur de romans historiques n'est pas le seul à avoir recours à l'imagination; l'historiographe le fait aussi, et beaucoup plus que ne le croient les gens en général. De même, le romancier est beaucoup moins libre qu'on le pense, car il ne peut rien mettre dans son œuvre qui soit en contradiction avec nos connaissances historiques. Erslev ne reconnaît finalement qu'une seule différence entre l'historiographie et le roman historique : on accorde au romancier, et à lui seul, le droit d'inventer des personnages fictifs à côté des personnages historiques et d'inventer des incidents, mettant éventuellement en jeu des personnages réels, que les sources ne connaissent pas. Mais aussitôt après avoir admis cette différence il s'applique à en minimiser la portée : le romancier ne peut rien inventer qui n'entre pas dans le cadre historique strict de la période qu'il envisage, tandis que l'historiographe peut de son côté extrapoler très librement à partir de ses sources. Ainsi, l'historien Erslev met le roman historique pratiquement au même niveau que l'historiographie, et ce rapprochement est fondamental : si l'on maintient que l'artiste et le poète s'occupent de ce qui est individuel et le scientifique de ce qui est général, dit-il, l'historien fait résolument partie des artistes. Et il se demande si les historiens accepteraient de reconnaître que le romancier a certains atouts qu'ils n'ont pas : par exemple l'intuition psychologique et la connaissance de l'homme.

Telle était donc l'atmosphère intellectuelle qui entourait Gunnar Gunnarsson lorsqu'il commençait à écrire sa série de romans historiques. Mais quelle était la situation de ces œuvres par rapport aux définitions de Kristian Erslev? La principale forme de romans historiques au XIX siècle était celle que Walter Scott avait créée. Dans cette " formule ", si l'on peut dire, le centre du roman est occupé par un personnage fictif, souvent assez médiocre en lui-même et peu intéressant (comme par exemple Waverley dans le roman du même nom) mais qui a la possibilité de passer d'un camp à un autre, dans une intrigue également fictive, et de réunir sous son regard différents aspects d'une situation historique. Celle-ci est en général un vaste mouvement venu des profondeurs du peuple, tel que le conflit entre la société clanique écossaise et la société «moderne » dans Waverley. Autour du personnage central gravitent d'autres personnages, beaucoup plus intéressants, qui prennent position et agissent mais qui restent dans leurs camps. Les grands personnages historiques apparaissent finalement à la périphérie et leur rôle se limite à leur véritable action historique, mais leur présence se fait sentir beaucoup plus que leurs apparitions ne le laissent penser ${ }^{3}$. C'est probablement à cette formule (utilisée entre autre, mais avec des modifications, par Johannes V.Jensen dans son célèbre roman La chute du roi, 1900-1901) que pensait Erslev lorsqu'il insistait sur la liberté du romancier de créer des personnages et des incidents. Il était entendu que ces fictions servaient essentiellement à mettre en valeur le contenu historique des romans, comme Walter Scott lui-même l'avait si bien fait.

10 Mais Gunnar Gunnarsson préférait suivre d'autres voies. Au lieu de mettre des personnages et des événements fictifs au centre de ses romans, il prenait des personnages historiques réels comme héros et utilisait comme trame ce que les sources nous apprennent sur les événements. Si l'on en croit Georges Lukâcs, c'est une gageure, car selon le philosophe hongrois il est quasiment impossible de mettre en scène dans un roman un véritable personnage historique de façon convaincante. Mais dans ses romans sur le Moyen âge, en tout cas, les sources sont suffisamment limitées pour permettre à Gunnar Gunnarsson de combiner un strict respect des faits et des 
personnages historiques et une grande liberté de création et d'interprétation. Dans Terre, où les sources dont il dispose sont tellement pauvres qu'il est obligé d'y suppléer en inventant des épisodes, il utilise d'ailleurs une formule originale qui remplace celle de Walter Scott : une grande partie des événements est vue par le regard d'un jeune enfant. Ainsi, les romans historiques de Gunnar Gunnarsson sont donc moins "romanesques ", pourrait-on dire, que les romans inspirés par la formule de Scott et encore plus proches de l'historiographie selon les définitions d'Erslev.

11 Pour écrire les deux romans en question, Les frères jurés et Terre, Gunnar Gunnarsson s'est très bien documenté. Dans Les frères jurés, où l'élément culturel joue un grand rôle, on voit que l'auteur connaît parfaitement tout ce qui avait été écrit à cette époque sur la civilisation des vikings. S'il prend plus de liberté dans Terre, nous savons que dans ce roman il a été fortement inspiré par une œuvre historico-philosophique, très connue à l'époque mais aujourd'hui tombée dans l'oubli: Notre peuple dans les temps anciens de Vilhelm Grønbech ${ }^{4}$. Mais plutôt que de voir les détails de sa documentation, il nous paraît important de voir ce qu'il a pu faire des «atouts» du romancier par rapport à l'historien, comme le disait Erslev. Après sa grande percée au Danemark avec le cycle romanesque L'histoire de la famille de Borg (1912-1914) et les romans " contemporains " qui suivaient, Gunnar Gunnarsson pouvait sans doute légitimement penser qu'il possédait la technique du roman, et on ne peut lire les deux romans historiques en question sans être frappé par sa capacité de dépeindre la psychologie des personnage et par sa qualité de visionnaire pour une période reculée. Mais il nous semble qu'il visait plus loin et qu'il voulait utiliser un autre « atout » du romancier que Kristian Erslev ne nomme pas mais qui est pourtant bien réel: celui d'envisager des problèmes que l'historien ne peut pas étudier, que ce soient les documents qui manquent ou la technique historique qui fasse défaut. C'est justement ce qu'il fait dans Les frères jurés et dans Terre et qui détermine la thématique de ces deux romans.

Gunnar Gunnarsson n'était pas le premier à soulever le problème qu'il traite dans ces deux romans. Cela avait été fait deux ans avant la publication des Frères jurés, mais d'une façon qui n'avait guère convaincu les historiens. En 1916, l'écrivain et l'historien norvégien Hans E. Kinck publia un article intitulé: "A propos des sagas. Des personnages qu'elles ne comprenaient pas $»^{5}$. La théorie qu'il propose dans ce texte célèbre est en soi peu défendable. Hans E. Kinck est convaincu que les sagas des Islandais, mises par écrit au XIII ${ }^{e}$ siècle mais dont l'action se situe entre le $\mathrm{IX}^{\mathrm{e}}$ et le début $\mathrm{du} \mathrm{XI} \mathrm{x}^{\mathrm{e}}$ siècle, racontent des événements historiques qu'on peut encore cerner même si ceux-ci ont été déformés par des générations de conteurs ou « sagnamenn ». L'une des raisons de ces déformations est, selon Kinck, l'interaction entre le public, qui veut des lignes claires et simples dans la narration, et le conteur qui observe les effets de son histoire sur l'auditoire placé devant lui et n'hésite pas à adapter le récit au goût de celui-ci. Du coup Kinck pense que certains personnages de sagas ont été mal compris ou déformés par les conteurs, qui ont mal interprétés leur caractère et leur actions, mais qu'il est possible de regarder derrière les textes et de découvrir la vérité. Parmi ces "personnages que les sagas ne comprenaient pas", il cite notamment Grímur-lechauve et son fils Egill dans la Saga d'Egill. Selon lui, leur comportement violent et leur besoin incessant d'activité s'expliquaient par le déracinement d'une famille de chefs qui avait été brutalement arrachée à sa patrie et obligée de s'installer ailleurs, et si la saga ne le dit pas, c'est parce que le conteur ou les générations de conteurs estimaient 
que le public ne comprendrait pas une telle explication et préféraient donc insister sur l'avidité des personnages et sur les exploits extérieurs ${ }^{6}$.

En expliquant des épisodes importants de certaines sagas par l'improvisation d'un conteur qui cherche à plaire à son auditoire, Hans E. Kinck fait parfois preuve d'une curieuse incompréhension pour la structure littéraire des textes qu'il a devant lui : on pourrait en effet lui reprocher d'ignorer les sagas telles qu'elles existent et d'inventer à leur place des romans de son propre cru. Même si les sagas proviennent probablement d'une longue tradition orale, tout leur contenu est intégré dans des élaborations complexes et un historien ne pourrait guère utiliser ces textes pour en tirer une quelconque conclusion sur des personnages réels au $\mathrm{IX}^{\mathrm{e}}$ ou au $\mathrm{x}^{\mathrm{e}}$ siècle. Mais il n'en va pas de même pour le romancier qui peut suivre d'autres voies que celles qu'il trouve directement dans les textes. Gunnar Gunnarsson avait probablement lu l'article de Hans E. Kinck - la description qu'il fait de Grímur-le-chauve et d'Egill dans Terre est tout à fait conforme à la théorie de l'écrivain norvégien - et là il pouvait justement trouver une question qui devait l'intéresser beaucoup, car elle était intimement liée à l'essence du mouvement "Blut und Boden", et qu'il avait peut-être déjà formulée : comment était-il possible pour des paysans norvégiens au $\mathrm{IX}^{\mathrm{e}}$ siècle, qui, malgré les expéditions viking, étaient fortement enracinés dans la terre de leurs ancêtres qu'ils connaissaient intimement et où ils avaient leurs lieux sacrés, de quitter brutalement cette terre et s'installer dans un pays neuf et inconnu, différent de tout ce qu'ils avaient connu auparavant et auquel rien ne les attachait? Et comment pouvaient-ils ensuite s'enraciner dans le nouveau pays?

Si Gunnar Gunnarsson choisit comme sujet des deux romans Les frères jurés et Terre, un vaste mouvement populaire, en accord avec la formule classique du roman historique, c'est-à-dire le peuplement d'un pays entier qui s'échelonne sur un demi-siècle, on trouve à la base de son projet littéraire ces deux questions, qui sont incontestablement pertinentes mais que l'historien est condamné à ignorer, et ce sont celles-ci qui déterminent la forme de ces œuvres. Ainsi, Gunnar Gunnarsson ne décrit pas le mouvement lui-même, - il ne fait que l'évoquer et n'explique qu'en passant les raisons politiques derrière toute cette émigration ${ }^{7}$. Même s'il souligne la continuité entre les deux romans en reprenant au début de Terre, mais sous un autre angle, un des épisodes finaux des Frères jurés, il concentre son attention pour ainsi dire exclusivement sur deux moments : le début et la fin du peuplement. Avec le début, l'arrivée du premier colon norvégien en Islande, il peut étudier la première question, celle du départ du pays ancestral et du déracinement, car c'est le moment où celle-ci se pose avec la plus grande clarté : le premier colon part pour des raisons d'ordre privé, il ne fait pas partie d'un mouvement (qui n'a pas encore commencé), il est donc pour ainsi dire seul et il arrive dans un pays inconnu et vide. Avec la fin, la création d'un parlement en Islande, l'auteur peut étudier la deuxième question, celle d'un nouvel enracinement.

Pour le premier moment, Gunnar Gunnarsson peut s'appuyer sur un passage du «Livre du peuplement ", Landnámabók, qui raconte d'une façon très succincte l'histoire du premier colon, Ingólfur Arnarson, et de son frère juré, Leifur, la raison de leur départ de la Norvège, leur arrivée en Islande, l'assassinat de Leifur par ses esclaves irlandais, et finalement l'installation d'Ingólfur à Reykjavík (l'endroit même où se trouve maintenant la capitale du pays) ${ }^{8}$. Ce texte, qui date sans doute du XIII ${ }^{e}$ siècle, lui fournit la trame des Frères jurés. Il le suit d'assez près, en brodant simplement sur les événements, les expéditions viking, les batailles etc., mais il ajoute deux choses : des 
éléments importants de l'histoire culturelle concernant la vie, la religion etc., et, surtout, la psychologie des personnages. Celle-ci est basée sur des indications données par les textes médiévaux, mais pour la souligner et la mettre en valeur, l'auteur ajoute des épisodes de son invention. La structure du roman est très claire, il est divisé en trois parties égales qui contiennent chacune douze chapitres.

16 1) La première partie, qui raconte l'enfance d'Ingólfur et de Leifur, est la plus originale. Sur cette partie de leur vie, le Landnámabók n'a rien à dire, ce texte explique simplement la parenté entre les deux personnages et précise qu'ils étaient aussi des «frères jurés ». L'auteur saisit cette idée de «fraternité jurée », il essaie d'imaginer les événements et les raisons psychologiques qui ont amené Ingólfur et Leifur à faire ce pas, et il termine par une superbe description d'une cérémonie religieuse païenne, lors de laquelle les deux amis deviennent des « frères jurés ».

2) C'est dans la deuxième partie que l'histoire commence réellement et là l'auteur suit Landnámabók de très près. Il raconte l'expédition viking d'Ingólfur et Leifur avec les trois fils du jarl Atli en Angleterre et en Irlande, la brouille qui suit, lorsqu'un des fils du jarl convoite Helga, la sœur d'Ingólfur et future femme de Leifur, l'expédition viking de Leifur seul l'été suivant, et la guerre ouverte entre les frères jurés et les fils d'Atli. Cette partie se termine par la condamnation à l'exil des frères jurés.

3) La troisième partie suit également le Landnámabók, mais avec quelques ajouts importants. L'auteur raconte d'abord le voyage d'exploration des deux frères jurés en Islande, qui vient d'être découverte et où ils ont décidé de s'installer, et leur retour en Norvège l'été suivant pour préparer leur départ définitif. Ensuite il raconte l'expédition viking de Leifur seul en Irlande, où il capture des esclaves et obtient une épée magnifique, mais sur l'acquisition de l'épée il invente une longue et importante histoire, très différente de celle qu'on trouve dans le Landnámabók. Après cela il décrit la navigation d'Ingólfur et Leifur vers l'Islande et leur séparation en mer, qu'il explique par un épisode de son invention. Sur l'assassinat de Leifur, l'auteur suit de nouveau le Landnámabók de près. Le roman se termine par l'installation d'Ingólfur et de sa famille à Reykjavik.

17 En elle-même cette histoire, que le Landnámabók racontait en quatre pages et que Gunnar Gunnarsson développe en quelque 200 pages, ne fournit aucune réponse à la question qui préoccupe l'auteur, à savoir comment il était possible de s'arracher à la terre des ancêtres, sans être déraciné, et de s'installer dans un nouveau pays. Mais un détail que donne le Landnámabók sans aucune explication met l'auteur sur la piste de la réponse. Leifur ne voulait jamais sacrifier aux dieux, alors qu'Ingólfur était très pieux, et après la mort de Leifur Ingólfur dit : «Il en va ainsi de quiconque ne veut pas faire des sacrifices». Pour que l'émigration puisse commencer, estime l'auteur, il fallait des conditions historiques particulières, à savoir la collaboration entre un "esprit d'aventure » et un " esprit d'enracinement », et il entreprend d'explorer dans le roman ces deux «esprits", leur coopération et leur destin, à partir du détail donné par le Landnámabók et à travers les deux personnages d'Ingólfur et de Leifur.

18 La personnalité d'Ingólfur apparaît très clairement dans les premières pages du roman. Dès son plus jeune âge il est fasciné par les images sculptées des dieux sur les montants du haut-siège : Odin avec ses corbeaux et Thor avec le marteau. Il croit en ces dieux et il les voit revivre le soir dans la lueur du feu du foyer. Si les statues dans le temple sont plus belles, ces images sculptées sont le centre de la famille, le signe visible de la continuité du lignage. Il sait que sur le haut-siège où son père est maintenant assis, il y 
avait son grand-père et ses ancêtres avant lui, et il sait aussi que plus tard il y sera assis à son tour, et ainsi de suite jusqu'au " crépuscule des dieux ». Ingólfur a un grand sens des responsabilités dès sa jeunesse, et il respecte toujours ses promesses. Lorsque son père vieillissant lui transmet l'administration des biens de la famille, il devient un dirigeant habile. Dans les expéditions vikings il est prudent et avisé.

La personnalité de Leifur est tout à fait à l'opposé. Il déteste les dieux, il ne croit pas en eux, il trouve les mythes stupides et, de plus, les sacrifices l'écœurent. Il est irresponsable et irréfléchi. S'il a une idée, il est obligé de la réaliser immédiatement et sans réflexion, quelle que soit l'absurdité de l'action. Il est incapable de tenir une promesse, car dès qu'il a une nouvelle idée il l'oublie. Sans arrêt il lui arrive des mésaventures de toutes sortes, et souvent il se met dans de graves dangers, - mais il s'en tire toujours. Lorsqu'on lui transmet l'administration des biens de sa famille, il s'en occupe bien pendant un moment mais il cesse de le faire lorsque cela ne l'amuse plus, de telle sorte qu'Ingólfur est obligé tacitement de prendre en charge ce travail. La vie quotidienne, ordinaire, ennuie profondément Leifur, et il prend les expéditions viking comme une sorte de libération. Dans les combats il est hardi mais imprudent. Ingólfur est obligé de le diriger, mais il peut aussi tirer profit de sa hardiesse.

Malgré cette profonde différence de caractère et des heurts occasionnels, Ingólfur et Leifur sont inséparables. Après leur condamnation à l'exil, Leifur ne peut plus penser à autre chose qu'à leur nouvelle destination. Il se demande sans arrêt où ils pourraient aller : tantôt il veut s'installer en Angleterre, tantôt en Irlande, aux Iles Féroé, en Shetland ou aux Orcades. Finalement il se souvient de ce pays qu'on vient de découvrir, l'Islande; il s'enflamme aussitôt pour cette idée, et il réussit à transmettre son enthousiasme à Ingólfur. Celui-ci est réceptif, car après la condamnation à l'exil, il a une nouvelle compréhension de sa situation. Il se rend maintenant compte que cet exil n'est pas totalement injuste : il avait guerroyé dans des pays étrangers et participé au pillage de gens contre lesquels il n'avait aucun grief. Ainsi il avait commis un crime contre ce qu'il y a de plus sacré, la Terre. Il comprend maintenant que la guerre est un mal, et il comprend aussi que le lignage qui n'a pas de terre où il peut pousser est condamné au malheur et à l'anéantissement. Il décide de ne plus utiliser son épée que pour se défendre.

21 Leifur a l'enthousiasme, mais il n'a pas cette nouvelle compréhension. Il se trouve bien le premier hiver en Islande, mais dès qu'il est en mer la vieille agitation le reprend et il continue les expéditions viking. Il capture des esclaves, des hommes innocents qui sont arrachés à leur propre pays, et il vole une épée magnifique à un ermite qui lui avait sauvé la vie. Ainsi, il a en fait prononcé sa propre condamnation. Mais il y a un dernier épisode capital. En s'approchant de l'Islande, Ingólfur veut que ce soit les dieux qui choisissent le lieu de sa ferme future: de cette façon uniquement il peut s'enraciner dans le nouveau pays. Il jette donc les montants du haut-siège à la mer en disant qu'il veut s'installer là où il les trouvera échoués. Jusqu'ici, le récit est conforme au Landnámabók, mais l'auteur ajoute une suite : Leifur réagit très violemment à ce qu'il estime être un acte de superstition stupide et il refuse de laisser des morceaux de bois décider de sa résidence. Les frères jurés se séparent donc et passent l'hiver dans des endroits séparés. Leifur est mal en point: il estime que ce séjour avec les esclaves irlandais, sans aucun espoir de nouvelles aventures, est vide de sens. Au printemps il est assassiné avec l'épée volée. 
Ainsi l'histoire est arrivée à son terme. Seul, Ingólfur ne serait jamais allé s'installer en Islande, et, seul, Leifur aurait été incapable de prendre la décision de se fixer définitivement dans un endroit. Pour que le peuplement de l'Islande puisse commencer il fallait la coopération des deux. Mais maintenant le rôle de Leifur est terminé. C'est lui qui a amené Ingólfur en Islande, mais en s'opposant à la décision d'Ingólfur de se soumettre à la volonté des dieux, il refuse en fait l'enracinement dans le nouveau pays, en tout cas l'enracinement que cherche Ingólfur. Sa mélancolie après le débarquement en Islande est symptomatique. Leifur doit disparaître, et il disparaît comme il a vécu. C'est ce qu'Ingólfur comprend à sa façon: il considère que Leifur était le véritable premier colon d'Islande mais que les dieux l'ont demandé en sacrifice. C'était un grand sacrifice, pense-t-il, mais ce n'est pas à lui de contester les décisions des dieux.

Après cela le peuplement de l'Islande peut commencer. Ingólfur trouve les montants du haut-siège dans la baie qu'il appelle Reykjavik et y établit sa ferme. Ainsi le pays n'est plus sauvage et vide, et d'autres peuvent suivre, bientôt poussés par les changements politiques en Norvège. Parmi eux il y a le dernier survivant des trois fils du jarlAtli, exilé à son tour par le roi Haraldur à la belle chevelure, qui est maintenant pleinement réconcilié avec Ingólfur.

Le roman Terrecommence par l'un des derniers épisodes des Frères jurés,l'arrivée d'Ingólfur à Reykjavik, qui est donc raconté ici pour la deuxième fois, et décrit ensuite les premières années de la famille dans ce nouveau lieu. Mais le lecteur voit ces événements de l'extérieur et d'une certaine distance : l'auteur ne décrit que ce qui est visible, et les personnages n'ont pas de nom et pas d'histoire antérieure. C'est comme si on décrivait les manifestations purement biologiques de la population, la seule force vitale. La véritable histoire commence ensuite au deuxième chapitre. Thorsteinn, le fils d'Ingólfur, est devenu un homme adulte, et sa femme est en train de donner naissance à leur premier fils. Ingólfur, très âgé, porte le nouveau-né dans le temple où il lui donne le nom de Thorkell Máni. Ensuite il transmet la charge de "prêtre principal » à son fils Thorsteinn et meurt cette même nuit. Thorsteinn commence aussitôt à préparer l'enterrement de celui qui est maintenant considéré comme le père du pays et il réunit ses nombreux amis dans toute cette partie sud de l'Islande, dont le lecteur fait maintenant connaissance.

Devenu le chef du lignage, Thorsteinn considère que c'est désormais son rôle de parachever l'œuvre que son père avait commencée, en réunissant les habitants du nouveau pays sous une loi unique et autour d'un parlement général. Les efforts qu'il fait pour cela fournissent la trame globale du roman, mais autour de ce sujet central se greffent beaucoup d'autres histoires plus ou moins bien développées, qui sont parfois rendues assez mystérieuses par le fait que de nombreux épisodes sont vus à travers le regard du jeune garçon Thorkell Máni.

Pour décrire les événements qui aboutirent à la fondation du parlement islandais à Thingvellir en 930, l'auteur n'a que des sources lacunaires, et la plupart des épisodes sont donc inventés par lui. Mais derrière le sujet politique central, il y a un autre thème encore plus profond: c'est la réponse à la deuxième question citée plus tôt, l'enracinement dans le nouveau pays. Ce thème central est présenté dès le début de l'histoire. Avant de mourir, Ingólfur dit à son deuxième fils : « Ne fais jamais confiance à un homme qui piétine la terre comme de la saleté - quelque haut qu'il pose son regard, Fils! Odin l'a embrassée et il l'embrasse toujours! Elle est féconde par sa grâce! »9. Lorsque Thora, la femme de Thorsteinn, voit comment Ingólfur meurt 
lorsque son petit-fils est né, elle a une nouvelle compréhension de la continuité de la vie, et elle cesse d'avoir peur de la mort.

A partir de là le thème se développe : c'est la terre et la vie humaine dans ce nouveau pays, en contact étroit avec la nature. Les habitants considèrent qu'ils forment une unité vitale avec la terre, ils ne sont pas les maîtres de la nature mais en forment une partie. Ainsi ils ont des facultés qui paraissent surnaturelles : trois frères, surnommés "les morses », peuvent par exemple attraper des oiseaux avec les mains et n'utilisent pas d'autres flèches que leur regard, un autre personnage, Björn-le-bouc, a fait alliance avec un être surnaturel vivant dans une falaise qui l'aide entre autre pour la pêche, etc. Le symbole de cette vie est la «danse de la forêt » de Thrasi qui imite la nature et les saisons ${ }^{10}$. Mais lors de l'enterrement d'Ingólfur, un autre personnage, Hrólfur à la barbe rousse, qui a trop bu, a une vision terrible qu'il exprime dans des vers du style de la «Prophétie de la voyante» (Völuspá).Ceux qui l'entendent n'y comprennent rien du tout, mais un lecteur $\mathrm{du} \mathrm{xx}^{\mathrm{e}}$ siècle devine qu'il a pu entrevoir la ville moderne à travers les vapeurs éthyliques... ${ }^{11}$

Lorsque Thorsteinn propose de créer un parlement général, il rencontre cependant une méfiance assez forte, et ses tentatives pour tenir un parlement régional ne réussissent pas très bien. Il a en effet des adversaires, qui sont cependant ses amis, et qui préfèrent une vie différente de celle que mènent Thorsteinn et ses voisins : ce sont les gens de Borg, Grímur-le-chauve et son fils Egill. Ils n'ont pas réussi à s'enraciner dans la nouvelle terre, ils ont rompu la loi du sang, sans arrêt inquiets, ils ne peuvent pas rester en place. Ce sont des poètes, des hommes de la parole, mais désorientés. Thorkell máni est horrifié par les descriptions de batailles que fait Egill. Parfois il ne peut pas s'empêcher de l'admirer, mais il sait une chose : s'il était comme Egill il mourrait à coup sûr.

Finalement, en faisant alliance avec des hommes qui ont la même idée que lui et en tirant astucieusement profit d'événements qui font apparaître la nécessité d'avoir une loi et des tribunaux, Thorsteinn réussit à accomplir l'œuvre de sa vie : la création d'un parlement général à Thingvellir. L'enracinement est devenu parfait. A la fin du roman, Thorkell Máni, dont on sait qu'il deviendra un chef politique important dans le pays, épouse une femme chrétienne: ainsi l'auteur annonce-t-il l'épisode suivant de l'histoire du pays, la christianisation, qu'il va décrire dans le roman Le Christ blanc.

\section{NOTES}

1. Cf. Halla Kjartansdóttir: "Í leit a eilífum sannindum. Um sögus鹥n Gunnars Gunnarssonar ", Andvari, Reykjavík, 1993, pp. 129-138.

2. Kristian Erslev: Historieskrivning. Grundlinier til nogle kapitler af historiens théorie, Copenhague, 1911.

3. La formule de Walter Scott, ou comme on dit « la forme classique du roman historique » a été analysée en particulier par Georges Lukács dans son étude Le roman historique, Paris, 1965. 
4. Vilhelm Grønbech: Vorfolkeœt i Oldtiden, Copenhague, 1909-1912. Cf. aussi l'article de Halla Kjartansdóttir (supra, n. 1).

5. Hans E. Kinck: «Et par ting om Ættesagaen. Skikkelser den ikke forstod », réimprimé dans Else Mundal: Sagadebatt, Oslo, 1977, pp. 164-186.

6. Ibidem, pp. 175-176.

7. Les sources médiévales expliquent ce mouvement d'émigration par les troubles provoqués par l'unification de la Norvège sous un roi unique, Haraldur à la belle chevelure, à la fin du $\mathrm{IX}^{\mathrm{e}}$ siècle. Les historiens modernes ont parfois mis en doute cette explication, mais Gunnar Gunnarsson l'acceptait comme le faisaient les historiens de son époque.

8. Des extraits importants du Landnámabók ont été traduits par Régis Boyer: Le livre de la colonisation de l'Islande (Landnámabók), Paris, 1973. Le passage sur Ingólfur et Leifur se trouve pp. 5-7.

9. Gunnar Gunnarsson : Jord, Copenhague, 1933, p. 25.

10. Ces descriptions sont influencées par les théories de Vilhelm Grønbech. Cf. Thröstur Helgason: "Eining og sundrung. Hinn form arfur í verkum Gunnars Gunnarssonar ", Lesbók Morgunbladsins, Reykjavík, 8 février 1997.

11. Gunnar Gunnarsson : Jord, Copenhague, 1933, p. 55-56.

\section{RÉSUMÉS}

L'article étudie deux romans historiques de l'écrivain islandais de langue danoise Gunnar Gunnarsson (1889-1975), Les frères jurés (Edbrødre, publié en 1918) et Terre (Jord, publié en 1933). Malgré la différence de style et d'esprit, ces romans forment un tout et traitent d'une période particulièrement importante dans l'histoire de l'Islande : la période du peuplement entre 874 et 930. L'auteur ne décrit pas ce mouvement dans son ensemble, il s'intéresse avant tout à deux moments : le début, avec l'installation du premier colon norvégien, qu'il décrit dans les Frères jurés, et la fin, avec l'établissement d'une société organisée, soumise à un parlement et une législation, qu'il décrit dans Terre. Pour illustrer le contexte intellectuel dans lequel ces deux romans furent écrits, l'article rappelle les théories de l'historien danois Kristian Erslev, publiées en 1911 : elles revendiquent l'autonomie de l'historiographie par rapport à la science historique et mettent le roman historique presqu'au même niveau que l'historiographie. Selon elles, le romancier historique a même des atouts que l'historien n'a pas. Les deux romans de Gunnar Gunnarsson paraissent tout à fait conformes aux théories d'Erslev, et les atouts du romancier ici consistent à envisager des problèmes historiques que l'historien est condamné à ignorer. Le problème qui intéresse Gunnar Gunnarsson avait déjà été posé indirectement par l'écrivain norvégien Hans E. Kinck en 1916 : c'est celui du déracinement des colons norvégiens, obligés de quitter brutalement la terre de leurs ancêtres, et leur enracinement dans un pays neuf. Dans ces deux romans, Gunnar Gunnarsson donne sa réponse : pour que le mouvement d'émigration fût possible il fallait une coopération particulière entre esprit d'aventure et esprit d'enracinement, l'un et l'autre personnifiés dans le premier roman par les deux frères jurés.

Im vorliegenden Aufsatz werden zwei historische Romane des isländischen Schriftstellers dänischer Sprache Gunnar Gunnarsson (1889-1975) untersucht, Eidbrüder (Edbrødre, erschienen 1918) und Erde (Jord, erschienen 1933). Trotz der Unterschiede in Stil und Geisteshaltung bilden die beiden Romane eine Einheit und behandeln einen für die Geschichte Islands besonders 
wichtigen Zeitabschnitt: die Periode der Besiedlung zwischen 874 und 930. Der Autor beschreibt diese Bewegung nicht in ihrer Gesamtheit, sondern er interessiert sich vor allem für zwei Zeitabschnitte: Den Beginn, mit der Ansiedelung des ersten norwegischen Siedlers, den er in Eidbruder beschreibt, und das Ende, gekennzeichnet durch die Errichtung einer organisierten Gesellschaft, in der die Gesetzgebung in einem Parlament erfolgt, das er in Erde beschreibt. Zur Beleuchtung des geistigen Hintergrunds dieser beiden Romane werden im Artikel die 1911 publizierten Theorien des dänischen Historikers Kristian Erslev angeführt: dieser fordert darin die Autonomie der Geschichtsschreibung gegenüber der Geschichtswissenschaft und stellt den historischen Roman fast auf dieselbe Ebene wie die Geschichtsschreibung. Demnach ist der Verfasser historischer Romane in gewissen Bereichen im Vorteil gegenüber dem Historiker. Die beiden Romane Gunnar Gunnarssons entsprechen offensichtlich ganz den Theorien Erslevs, und die Stärke des Autors scheint hier die Berücksichtigung einer historischen Problematik zu sein, die der Historiker nicht beachten kann. Die Probleme, für die sich Gunnar Gunnarsson interessiert, wurden indirekt schon vom norwegischen Schriftsteller Hans E. Kinck 1916 behandelt, nämlich die Entwurzelung der norwegischen Siedler, die plötzlich das Land ihrer Ahnen verlassen müssen und ihre Verwurzelung in einem neuen Land. Gunnar Gunnarsson gibt in diesen beiden Romanen seine Antwort darauf: eine Auswanderungsbewegung ist nur durch das Zusammenspiel von Abenteuerlust und Bodenständigkeit möglich, beide personifiziert im ersten Roman durch die zwei Eidbrüder.

\section{AUTEUR}

\section{EINAR MÁR JÓNSSON}

Université de Paris IV 